\title{
Avaliação da eficácia e efetividade de treinamentos ministrados à enfermagem de um hospital geral acreditado
}

\begin{abstract}
RESUMO | Objetivos: Analisar o processo de avaliação da reação, aprendizagem, comportamento e de resultados dos treinamentos ministrados à equipe de enfermagem. Método: Tratou-se de um estudo descritivo, exploratório, de campo de abordagem quantitativa. Resultados: Na avaliação de reação, verificou-se que 75,0\% apontaram como adequadíssimas a atuação do facilitador e 64,3\% à estratégia adotada. Na avaliação de aprendizagem verificou-se um aumento do número de acertos apenas na questão nove cuja porcentagem passou de $25,0 \%$ para $71,4 \%$. Quanto à avaliação de comportamento, não foram verificadas mudanças no padrão de comportamento das anotações de enfermagem de todos os três itens entre a primeira e a segunda avaliação e entre a segunda e terceira avaliação. Quanto à avaliação de resultados foi verificado um aumento na média de anotações corretas para oito itens. Conclusão: Frente ao resultado deste estudo, considera-se necessária a responsabilização dos enfermeiros no processo de desenvolvimento da equipe.
\end{abstract}

Palavras-chaves: Enfermagem; Treinamento; Educação Continuada em Enfermagem; Avaliação; Avaliação Educacional.

\begin{abstract}
Objectives: To analyze the process of evaluating the reaction, learning, behavior and results of the training given to the nursing team. Method: This was a descriptive, exploratory, field study with a quantitative approach. Results: In the reaction assessment, it was found that $75.0 \%$ indicated the role of the facilitator as very adequate and $64.3 \%$ to the adopted strategy. In the learning assessment, there was an increase in the number of correct answers only in question nine, whose percentage went from $25.0 \%$ to $71.4 \%$. As for the behavioral assessment, there were no changes in the behavior pattern of the nursing notes of all three items between the first and the second assessment and between the second and the third assessment. As for the evaluation of results, there was an increase in the average of correct notes for eight items. Conclusion: In view of the result of this study, it is considered necessary to hold nurses accountable in the team development process.
\end{abstract}

Keywords: Nursing; Training; Continuing Education in Nursing; Evaluation; Educational Evaluation.

RESUMEN | Objetivos: Analizar el proceso de evaluación de la reacción, el aprendizaje, el comportamiento y los resultados de la capacitación impartida al equipo de enfermería. Método: Este fue un estudio de campo descriptivo, exploratorio, con un enfoque cuantitativo. Resultados: En la evaluación de la reacción, se encontró que el $75.0 \%$ indicó que el rol del facilitador era muy adecuado y el $64.3 \%$ a la estrategia adoptada. En la evaluación del aprendizaje, hubo un aumento en el número de respuestas correctas solo en la pregunta nueve, cuyo porcentaje pasó del $25.0 \%$ al $71.4 \%$. En cuanto a la evaluación del comportamiento, no hubo cambios en el patrón de comportamiento de las notas de enfermería de los tres ítems entre la primera y la segunda evaluación y entre la segunda y la tercera evaluación. En cuanto a la evaluación de resultados, hubo un aumento en el promedio de notas correctas para ocho ítems. Conclusión: en vista del resultado de este estudio, se considera necesario responsabilizar a las enfermeras en el proceso de desarrollo del equipo.

Descriptores: Enfermería; Formación; Educación Continua en Enfermería; Evaluación; Evaluación Educativa.

\section{Paula Maria Corrêa de Gouveia Araujo}

Enfermeira, Mestre em Ciências da Saúde pela Escola Paulista de Enfermagem-UNIFESP. Supervisora Enfermagem responsável pela Educação Continuada e Núcleo de Segurança do Paciente do Hospital Santa Marcelina de Itaquaquecetuba. Membro do Grupo de Estudo e Pesquisa em Avaliação dos Serviços de Saúde e Enfermagem (GEPAV-SE) /EPE - UNIFESP.

\section{Maria D' Innocenzo}

Enfermeira, doutora em ciências da saúde. Doutora, professora associada Escola Paulista de Enfermagem / UNIFESP. Coordenadora do Grupo de Estudo e Pesquisa em Avaliação dos Serviços de Saúde e Enfermagem (GEPAV-SE) /EPE - UNIFESP.

Recebido em: 15/02/2020

Aprovado em: 17/02/2020

\section{Maria Lucia Alves Pereira Cardoso}

Enfermeira, Especialista em Gestão de Saúde em Enfermagem e Doutora em Ciências pela Escola Paulista de Enfermagem - UNIFESP. Docente e Coordenadora de Pós Graduação da FECS Faculdade do Hospital Alemão Oswaldo Cruz. Membro do Grupo de Estudo e Pesquisa em Avaliação dos Serviços de Saúde e Enfermagem (GEPAVSE) / EPE - UNIFESP.
INTRODUÇÃO

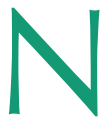
as Instituições prestadoras de serviços de saúde, a equipe de enfermagem constitui o maior número de profissionais, atuando na assistência à saúde, contingente este estimado em $60 \%$. Desse modo, o sucesso e efetividade na formação e capacitação desses profissionais estão diretamente relacionados aos resultados positivos obtidos com as melhores práticas assistenciais ${ }^{(1)}$.

$\mathrm{O}$ interesse pelo tema relativo à avaliação de treinamentos ministrados à equipe de enfermagem tornou-se imprescindível, visto que se trata de um assunto de suma importância, pois o grande desafio não é só ministrar ou facilitar que ocorram as atividades educativas, mas sim mensurar a eficácia e efetividade dos treinamentos, através da verificação do 
aprendizado, da mudança de comportamento e do impacto nos resultados.

Treinamento é uma das estratégias para aquisição de aprendizagem. Pode ser definido como "um processo educacional que é aplicado de maneira sistemática e organizada pelo qual as pessoas adquirem conhecimentos, atitudes e habilidades em decorrência dos objetivos definidos"(2). Modernamente, é considerado um meio para melhorar o desempenho no cargo $^{(3)}$.

Desta forma, para prestar uma assistência qualificada ao cliente, atendendo às novas exigências do mercado de trabalho e do desenvolvimento tecnológico das empresas, estas precisam desenvolver estratégias para atualização contínua dos seus profissionais ${ }^{(4,5)}$. Na área da saúde devem ser intensificadas as ações educativas para garantir a segurança dos profissionais, dos pacientes e da instituição.

Cabe destacar que é necessário o alinhamento do treinamento com as diretrizes corporativas e também com o ambiente no qual a empresa está inserida ${ }^{(6,7)}$.

As empresas investem cada vez mais em treinamentos com o objetivo de que os participantes aprendam e transfiram novos conhecimentos, habilidades e atitudes para o seu ambiente de trabalho(4). Entende-se que esse seja o maior desafio, transferir e aplicar o conhecimento na prática.

O treinamento é um processo cíclico e continuo composto de quatro etapas: Levantamento das necessidades de treinamento (LNT), Programação de treinamento, Implementação e Execução do programa e Avaliação dos resultados ${ }^{(8)}$.

Por meio da avaliação dos processos educativos, será possível, ainda, obter indicadores que demonstrem em ações práticas os resultados desses processos ${ }^{(9)}$. A avaliação de treinamentos é capaz de proporcionar, no minimo, um importante indicador de uso dos recursos financeiros e não financeiros da organização ${ }^{(10)}$.

Esta etapa tem a finalidade de verificar a efetividade das ações instrucionais ${ }^{(11)}$, ou seja, constatar se após certo período da aplicação do treinamento há evidencias de aprendizado.
Ao se avaliar um treinamento devem-se levar em consideração todos os passos adotados para o planejamento do mesmo ${ }^{(11)}$.

É comum, nos serviços de saúde, a prática de programas educacionais, porém percebem-se dificuldades em encontrar exemplos de avaliações de seus resultados ${ }^{(12)}$.

Nesse estudo, o referencial teórico utilizado foi de Donald Kirkpatrick, pois além de ser pioneiro e consagrado internacionalmente, é considerado exemplo tradicional da área de treinamento com foco em resultado e o seu modelo contribui com os objetivos deste estudo.

Nos anos 1950, Donald Kirkpatrick publicou artigos, nos quais estabelecia quatro níveis de avaliação: avaliação de reação, avaliação de aprendizagem, avaliação de comportamento e avaliação de resultado ${ }^{(13)}$. A seguir são apresentados os quatro níveis estabelecidos por Donald Kirkpatrick:

Nível 1 - Reação mede a satisfação dos participantes com o treinamento;

Nível 2 - Aprendizagem, diz respeito à melhora no conhecimento, habilidade e atitude dos participantes proporcionada pela participação nos treinamentos;

Nível 3 - Comportamento, refere-se a mudança de atitude em função da participação em um programa de treinamento;

Nível 4 - Resultados, reflete o retorno para a organização, da participação do profissional em programas de treinamento, podendo ser medido em termos de aumento de produção, melhora da qualidade, redução de custos, menor rotatividade, melhor lucro e menor frequência ou gravidade de acidentes ${ }^{(14)}$.

Embora muitos profissionais identifiquem a importância do assunto, poucos estudos apontam a validação dos treinamentos realizados nas instituições de saúde.

Os objetivos deste estudo foram: analisar o processo de avaliação da reação, no que se refere à metodologia utilizada, local, facilitador, material e horário dos treinamentos ministrados à equipe de enfermagem; analisar o processo de avaliação da aprendizagem dos treinamentos ministrados à equipe de enfermagem; analisar o processo de avaliação de comportamento dos treinamentos ministrados à equipe de enfermagem; analisar o processo de avaliação de resultados dos treinamentos.

\section{METODOLOGIA}

Para alcançar os objetivos propostos neste estudo, optou-se em utilizar o método descritivo correlacional, exploratório, de campo sob a abordagem quantitativa.

$\mathrm{Na}$ coleta de dados, foi utilizada a descrição das técnicas citadas a seguir, visto que a coleta tem a finalidade de obter informações para que os objetivos sejam alcançados.

Estas técnicas usadas foram: Observação e Pesquisa documental.

A observação é uma técnica que usa os sentidos para obtenção de determinados aspectos da realidade ${ }^{(15)}$.

$\mathrm{Na}$ pesquisa documental, a fonte de coleta de dados é restrita a documentos escritos ou não ${ }^{(15)}$.

O estudo foi desenvolvido em uma Organização Social de Saúde (OSS), de parceria com Governo Estadual. Trata-se de um hospital geral de nível terciário, sendo também centro de referência para o trauma, localizado na região de Itaquaquecetuba da Grande São Paulo. Esta Unidade foi inaugurada em março de 2000, possui 247 leitos, dispõe de elevada estrutura para atender os 11 municípios da região.

Os setores selecionados para a realização do estudo de campo foram: a Maternidade (alojamento conjunto) e Unidade Neonatal (Berçário Patológico) do referido Hospital, por estarem relacionados à formação da pesquisadora, por possuírem protocolos específicos e ser referência para a região pelo atendimento de casos de alto risco.

A população em estudo foi representada pelos profissionais auxiliares de enfermagem que atuam a mais de 90 dias na Maternidade (alojamento conjunto) e Unidade Neonatal da instituição onde o estudo foi realizado, e os critérios de exclusão foram: profissionais que estavam de férias, folga, licença-maternidade, licença médica e os admitidos ou rema- 
nejados para o setor nos últimos 90 dias antes da aplicação do treinamento.

Neste estudo, os instrumentos foram desenvolvidos através da experiência da pesquisadora e por meio de uma revisão da literatura com enfoque no modelo da avaliação de treinamentos do Donald Kirkpatrick e recomendações sobre anotação de enfermagem do Conselho Regional de Enfermagem de São Paulo (COREN SP).

Antes de iniciar a coleta de dados, os instrumentos foram encaminhados para avaliação do conteúdo. Foi utilizada a técnica de Delphi para verificação da clareza e da relevância do conteúdo das afirmativas dos instrumentos de pesquisa.

A coleta de dados foi realizada depois de concluída as $1^{\mathrm{a}}, 2^{\mathrm{a}}$ e $3^{\mathrm{a}}$ etapas descritas a seguir:

$\checkmark \quad 1^{\text {a }}$ etapa: apreciação e aprovação da Gerência de Enfermagem e Diretoria Administrativa do Hospital campo de estudo, localizado em Itaquaquecetuba. (Anexo 1).

$\checkmark \quad 2^{a}$ etapa: apreciação e aprovação do Termo de Consentimento Livre e Esclarecido - TCLE (Anexo 2) pelo Comitê de Ética em Pesquisa da Universidade Federal de São Paulo (UNIFESP) - Projeto n. ${ }^{\circ}$ 0019/2015, Parecer: n. ${ }^{\circ}$ 991.271, n. ${ }^{\circ}$ CAAE: 4065 3914.5.0000.5505. Após foi feita a revalidação pelo Comitê de Ética em Pesquisa da Instituição localizada em Itaquera - SP, que também pertence à rede do Hospital do estudo de Itaquaquecetuba.

$\checkmark \quad 3^{\text {a }}$ etapa: aplicação do TCLE.

O participante do estudo foi abordado individualmente em uma sala do Hospital de Itaquaquecetuba no período de 09 a 11/03/2016 onde recebeu informações sobre o objetivo e a finalidade do trabalho.

Depois de esclarecidas as dúvidas, o convidado que aceitou o convite, assinou o Termo, dando seu consentimento livre e esclarecido na presença do pesquisador.

Os dados foram coletados em três etapas.

A primeira etapa ocorreu 30 dias antes do treinamento no período de 17/03/16 a 23/03/16 com a realização da análise dos registros de enfermagem pela pesquisadora utilizando o instrumento de Avaliação Resultados - nível 4. Na Instituição, local de estudo havia um cronograma de treinamento com a previsão da abordagem do tema Anotação de Enfermagem. A partir daí foi elaborado o Programa de treinamento.
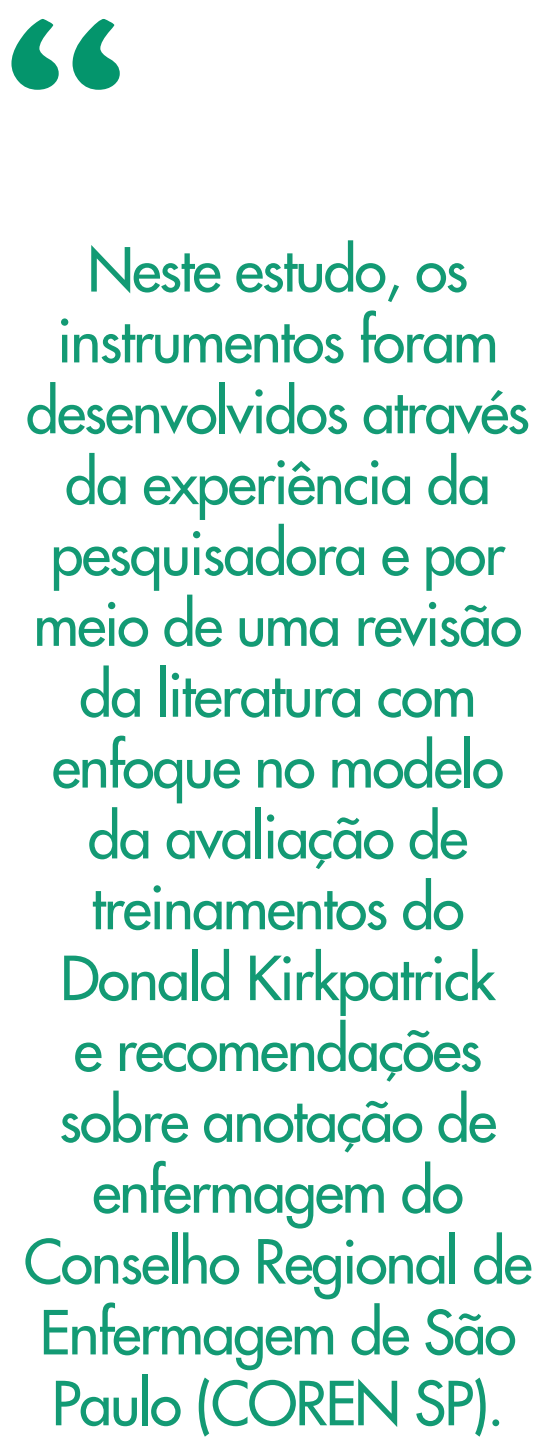

A segunda etapa correspondeu ao momento de aplicação do treinamento, que ocorreu de 26/04 a 01/05/16. As estratégias utilizadas para o treinamento foram aula teórica e exemplos de anotações de enfermagem, retirados de prontuários dos pacientes. O treinamento ocorreu em diversos horários: no início do plantão às 7 h10 e 13h10, no meio do plantão às $15 \mathrm{~h}$ e ao término do plantão às $5 \mathrm{~h} 30$ com duração aproximada de 30 minutos cada abordagem. O material didático utilizado se constituiu de apostila em Power Point, guia de anotação de enfermagem e cases. Todos os treinamentos foram realizados no local de trabalho dos participantes do treinamento, nas Unidades: Neonatal e Maternidade - Alojamento Conjunto.

A facilitadora de todos os treinamentos foi a enfermeira responsável pelo setor de Educação Continuada do Hospital da realização do estudo. Antes do início do treinamento foi entregue o impresso de caracterização do sujeito e o instrumento de avaliação de aprendizagem para que os profissionais respondessem. Os treinamentos foram aplicados nas datas e locais previstos, com exceção de um único agendamento que foi reprogramado devido à intercorrência no setor. Em todas as abordagens a lista de frequência padronizada pela Instituição foi assinada pelos participantes para que ficasse registrada a presença ao treinamento. Ao término do treinamento foi solicitado novamente aos participantes que respondessem a avaliação de aprendizagem.

A terceira etapa realizada 30 dias após a aplicação do treinamento no período de 24/05 a 13/06/16. Esta fase contemplou o preenchimento da avaliação de comportamento (nível 3) pelo gestor imediato do auxiliar de enfermagem que participou do treinamento e a análise dos registros de enfermagem pela pesquisadora com a utilização do instrumento (Avaliação Resultados - nível 4).

Os dados coletados foram organizados e armazenados em planilhas eletrônicas, utilizando o software Microsoft Excel $\AA$. Posteriormente, os dados foram analisados descritivamente. Para as variáveis categóricas foram apresentadas frequências absolutas e relativas e para as variáveis numéricas, medidas-resumo (média, quartis, mínimo máximo e desvio padrão). 
Tabela 1. Distribuição da Avaliação de reação ao treinamento ministrado. São Paulo, SP, Brasil, 2016

\begin{tabular}{|c|c|c|}
\hline Reação & $\mathrm{N}$ & $\%$ \\
\hline Estratégias de ensino & 28 & 100,0 \\
\hline Adequadíssimo & 18 & 64,3 \\
\hline Bem adequado & 7 & 25,0 \\
\hline Adequado & 3 & 10,7 \\
\hline Horário & 28 & 100,0 \\
\hline Adequadíssimo & 7 & 25,0 \\
\hline Bem adequado & 10 & 35,7 \\
\hline Adequado & 6 & 21,4 \\
\hline Pouco adequado & 4 & 14,3 \\
\hline Não adequado & 1 & 3,6 \\
\hline Material Didático & 28 & 100,0 \\
\hline Adequadíssimo & 14 & 50,0 \\
\hline Bem adequado & 9 & 32,1 \\
\hline Adequado & 5 & 17,9 \\
\hline Local & 28 & 100,0 \\
\hline Adequadíssimo & 12 & 42,9 \\
\hline Bem adequado & 9 & 32,1 \\
\hline Adequado & 6 & 21,4 \\
\hline Pouco adequado & 1 & 3,6 \\
\hline Facilitador & 28 & 100,0 \\
\hline Adequadíssimo & 21 & 75,0 \\
\hline Bem adequado & 6 & 21,4 \\
\hline Adequado & 1 & 3,6 \\
\hline
\end{tabular}

\section{RESULTADOS}

$\mathrm{Na}$ Tabela 1 pode-se observar que $21(75,0 \%)$ sujeitos que participaram da pesquisa assinalaram a escala Likert utilizada na avaliação de reação como sendo adequadíssimo a atuação do facilitador, $18(64,3 \%)$ a estratégia de ensino utilizada, 14 (50,0\%) o material didático e 12 $(42,9 \%)$ o local da realização do treinamento e apenas 7 (25\%) o horário de realização do treinamento.

\section{DISCUSSÃO}

O objetivo da avaliação de reação é investigar se o treinando está satisfeito, ou seja, se tem reação favorável em relação ao evento que acabou de participar ${ }^{(16)}$.

Estudo realizado aponta que o profissional sente-se mais seguro em um ambiente que lhe é familiar, como o seu local de trabalho ${ }^{(17)}$. Mas não foi o que ocorreu nesta pesquisa conforme os resultados apresentados na Tabela1. Pode-se inferir que isso se sucedeu devido ter sido reunida toda a equipe de auxiliares de enfermagem do setor para participar no mesmo momento, é possível que se tivesse dividido em turmas, as participantes ficariam mais tranquilas e com a atenção voltada exclusivamente para o treinamento.

Tabela 2. Distribuição do percentual de respostas certas e erradas nos momentos pré e pós-treinamento. São Paulo, SP, Brasil, 2016

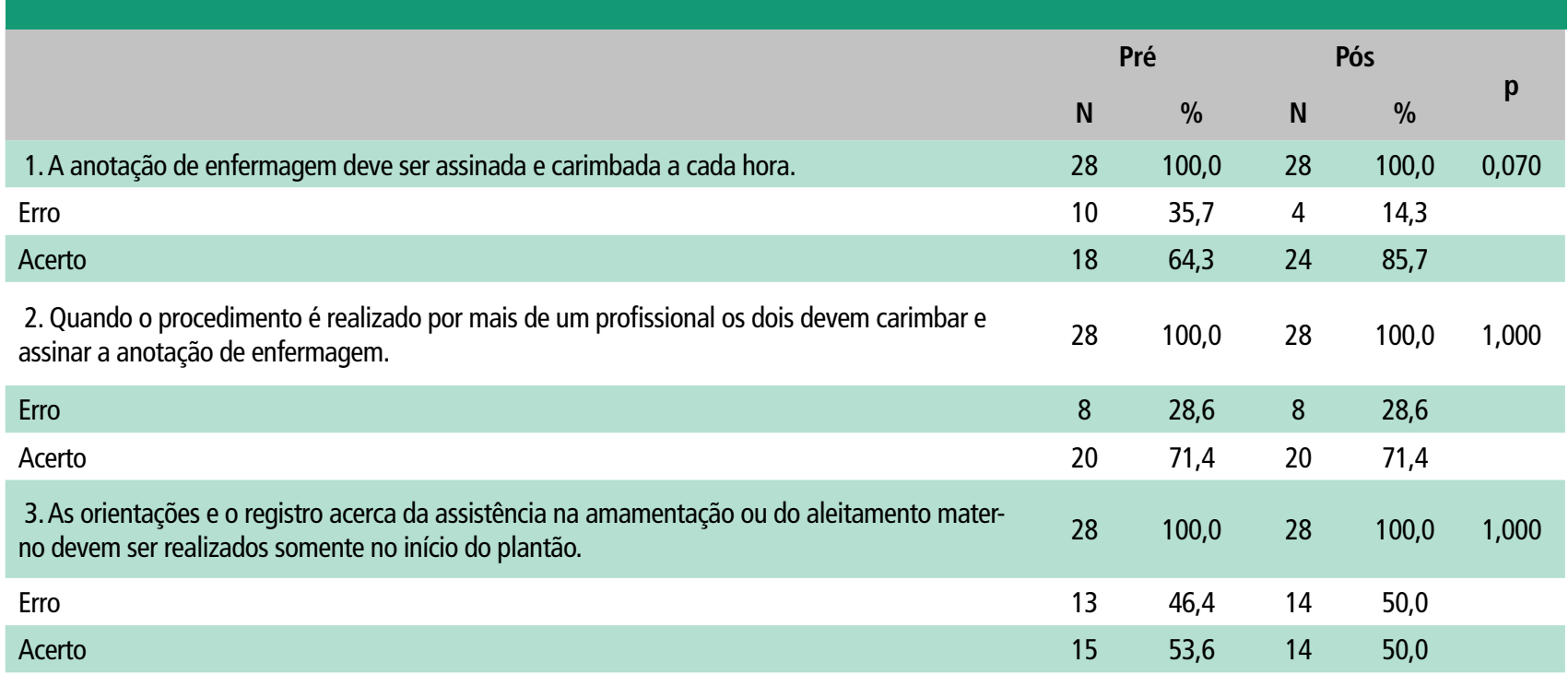


Araujo, P.M.C.G.; D' Innocenzo, M.; Cardoso, M.L.A.P.;Avaliação da eficácia e efetividade de treinamentos ministrados à enfermagem de um hospital geral acreditado

4. É necessário registrar a pega e a sucção ao seio materno (presença ou ausência) e características da sucção (efetiva ou inadequada) sempre após as mamadas.

\begin{tabular}{|c|c|c|c|c|c|}
\hline Erro & 5 & 17,9 & 3 & 10,7 & \\
\hline Acerto & 23 & 82,1 & 25 & 89,3 & \\
\hline $\begin{array}{l}\text { 5. É importante realizar a anotação de enfermagem após orientar a mãe para manter o recém- } \\
\text {-nascido no berço em decúbito dorsal. }\end{array}$ & 28 & 100,0 & 28 & 100,0 & 0,289 \\
\hline Erro & 9 & 32,1 & 5 & 17,9 & \\
\hline Acerto & 19 & 67,9 & 23 & 82,1 & \\
\hline Erro & 23 & 82,1 & 17 & 60,7 & \\
\hline Acerto & 5 & 17,9 & 11 & 39,3 & \\
\hline $\begin{array}{l}\text { 7. A anotação de enfermagem deve ser realizada logo após a ocorrência de uma intercorrência } \\
\text { com a mãe ou recém-nascido. }\end{array}$ & 28 & 100,0 & 28 & 100,0 & 0,375 \\
\hline $\begin{array}{l}\text { 8. Uma das finalidades da anotação de enfermagem é servir de documento de proteção para o } \\
\text { profissional de enfermagem. }\end{array}$ & 28 & 100,0 & 28 & 100,0 & 1,000 \\
\hline Erro & 2 & 7,1 & 1 & 3,6 & \\
\hline Acerto & 26 & 92,9 & 27 & 96,4 & \\
\hline $\begin{array}{l}\text { 9. As anotações de enfermagem devem ser finalizadas com as frases: "sem intercorrências", } \\
\text { "segue aos cuidados da enfermagem". }\end{array}$ & 28 & 100,0 & 28 & 100,0 & 0,002 \\
\hline Erro & 21 & 75,0 & 8 & 28,6 & \\
\hline Acerto & 7 & 25,0 & 20 & 71,4 & \\
\hline $\begin{array}{l}\text { 10. Os sinais vitais devem ser registrados na anotação de enfermagem imediatamente após a } \\
\text { conferência. }\end{array}$ & 28 & 100,0 & 28 & 100,0 & - \\
\hline Acerto & 27 & 96,4 & 27 & 96,4 & \\
\hline 12. Se o profissional estiver sem o carimbo, deve colocar o primeiro nome, $\mathrm{RG}$ e $\mathrm{n}^{\circ}$ do Conselho. & 28 & 100,0 & 28 & 100,0 & 1,000 \\
\hline Erro & 22 & 78,6 & 21 & 75,0 & \\
\hline Acerto & 6 & 21,4 & 7 & 25,0 & \\
\hline
\end{tabular}

Notas: p- Nível descritivo do teste de McNemar.

(-). Não foi possível realizar o teste de McNemar - Ausência de acerto na avaliação inicial

Tabela 3. Distribuição de percentuais de acertos e erros em relação ao comportamento adotado pelos auxiliares de enfermagem nos momentos de aprendizagem. São Paulo, SP, Brasil, 2016

\section{1a. Avaliação $\quad$ 2a. Avaliação $\quad 3 a$. Avaliação}

p

1. As anotações de enfermagem foram realizadas logo após o recebimento do plantão

$\begin{array}{cccccccc}\mathbf{N} & \% & \mathbf{N} & \% & \mathbf{N} & \% & \begin{array}{c}\text { 1a. Aval. } \mathbf{x} \\ \text { 2a. Aval. }\end{array} & \begin{array}{c}\text { 2a. Aval. } \mathbf{x} \\ \text { 3a. Aval. }\end{array} \\ 28 & 100,0 & 28 & 100,0 & 28 & 100,0 & 1,000 & 1,000 \\ 22 & 78,6 & 22 & 78,6 & 22 & 78,6 & & \end{array}$




\begin{tabular}{llllllllll}
$\begin{array}{l}\text { 3. Na anotação de enfermagem no inicio do plantão está especi- } \\
\text { ficado que o Rn / Puérpera mantêm pulseira de identificação }\end{array}$ & 28 & 100,0 & 28 & 100,0 & 28 & 100,0 & 0,500 & 1,000 \\
\hline Erro & 17 & 60,7 & 19 & 67,9 & 18 & 64,3 & & \\
\hline Acerto & 11 & 39,3 & 9 & 32,1 & 10 & 35,7 &
\end{tabular}

Tabela 4. Distribuição das medidas-resumo do número de anotações de enfermagem corretas na avaliação de resultados. São Paulo, SP, Brasil, 2016

Média $\begin{gathered}\text { Desvio } \\ \text { Padrão }\end{gathered}$ Mínimo Máximo $\begin{gathered}1^{\circ} \\ \text { Quartil }\end{gathered}$ Mediana $\begin{gathered}3^{\circ} \\ \text { Quartil }\end{gathered} \quad$ N $\quad$ p

Segurança

1.1. Apresenta registro sobre a presença da pulseira de identificação no paciente.

\begin{tabular}{llllllllll} 
Pré & 2,33 & 0,87 & 0,00 & 3,00 & 2,00 & 3,00 & 3,00 & 24 \\
\hline Pós & 2,83 & 0,64 & 0,00 & 3,00 & 3,00 & 3,00 & 3,00 & 24 \\
\hline Diferença (Pós- Pré) & 0,50 & 0,83 & $-1,00$ & 3,00 & 0,00 & 0,00 & 1,00 & 24
\end{tabular}

1.2. Na anotação de enfermagem há registro de que foi realizada a dupla checagem na administração de drogas de alerta

\begin{tabular}{|c|c|c|c|c|c|c|c|c|c|}
\hline $\begin{array}{l}\text { 1.3. Apresenta registro de pelo menos duas } \\
\text { medidas de prevenção de queda do RN } \\
\text { (Orientação para mãe transportar Rn no ber- } \\
\text { ço e não dormir com o Rn no colo; manter } \\
\text { portinhola fechada da incubadora) }\end{array}$ & & & & & & & & & 0,001 \\
\hline Pré & 0,29 & 0,56 & 0,00 & 2,00 & 0,00 & 0,00 & 0,50 & 21 & \\
\hline Pós & 1,43 & 1,29 & 0,00 & 3,00 & 0,00 & 2,00 & 3,00 & 21 & \\
\hline Diferença (Pós- Pré) & 1,14 & 1,28 & $-1,00$ & 3,00 & 0,00 & 1,00 & 2,00 & 21 & \\
\hline \multicolumn{10}{|l|}{ Administração do leite } \\
\hline $\begin{array}{l}\text { 2.1. Apresenta registro sobre a presença } \\
\text { ou não de sucção ao seio materno e a sua } \\
\text { característica (efetiva ou insuficiente) }\end{array}$ & & & & & & & & & 0,001 \\
\hline Pré & 0,53 & 0,83 & 0,00 & 2,00 & 0,00 & 0,00 & 1,00 & 15 & \\
\hline Pós & 1,93 & 1,03 & 0,00 & 3,00 & 1,00 & 2,00 & 3,00 & 15 & \\
\hline Diferença (Pós- Pré) & 1,40 & 1,24 & $-1,00$ & 3,00 & 1,00 & 1,00 & 3,00 & 15 & \\
\hline
\end{tabular}

2.2. Apresenta anotação de orientação à mãe sobre: vantagens do aleitamento materno, frequência da amamentação, tempo de cada mamada, pega correta, higienização das mãos e incentivo à ingesta hídrica

\begin{tabular}{|llllllllll} 
Pré & 0,20 & 0,56 & 0,00 & 2,00 & 0,00 & 0,00 & 0,00 & 15 \\
\hline Pós & 1,47 & 1,19 & 0,00 & 3,00 & 0,00 & 1,00 & 3,00 & 15 \\
\hline Diferença (Pós- Pré) & 1,27 & 1,33 & $-1,00$ & 3,00 & 0,00 & 1,00 & 3,00 & 15
\end{tabular}

2.3. Apresenta anotação referente orientação sobre o posicionamento da puérpera e Rn para a amamentação.

$\begin{array}{lllllllll}\text { Pré } & 0,00 & 0,00 & 0,00 & 0,00 & 0,00 & 0,00 & 0,00 & 15 \\ \text { Pós } & 1,27 & 1,16 & 0,00 & 3,00 & 0,00 & 1,00 & 2,00 & 15\end{array}$


Araujo, P.M.C.G.; D' Innocenzo, M.; Cardoso, M.L.A.P.; Avaliação da eficácia e efetividade de treinamentos ministrados à enfermagem de um hospital geral acreditado

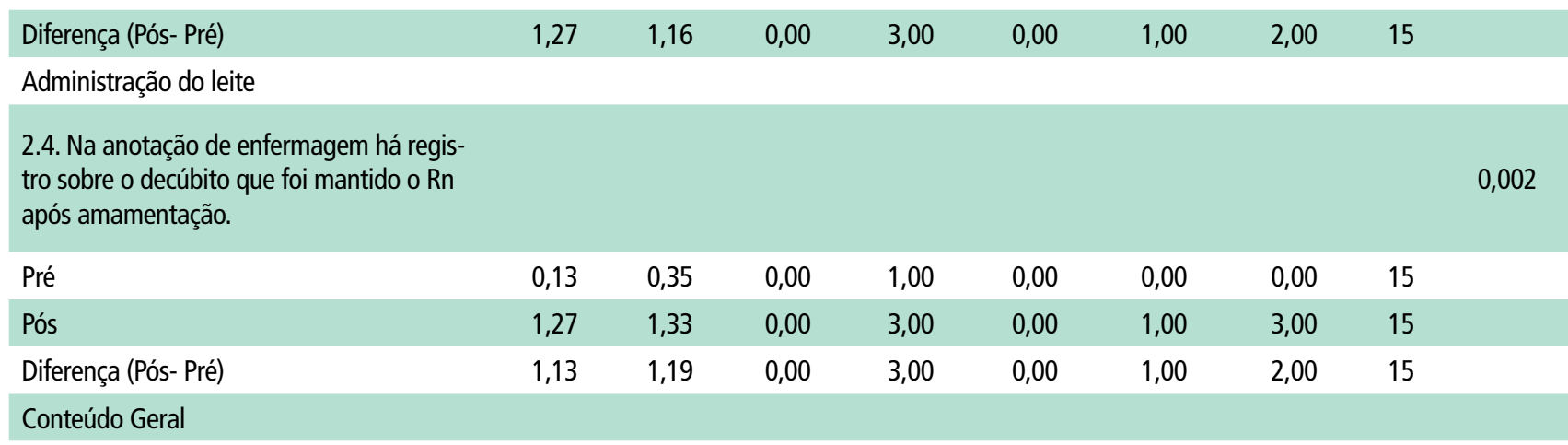

3.1. Consta carimbo e assinatura do profissional de enfermagem ao final da anotação de enfermagem

\begin{tabular}{|llllllllll|}
\hline Pré & 2,30 & 1,07 & 0,00 & 3,00 & 2,00 & 3,00 & 3,00 & 27 \\
\hline Pós & 2,93 & 0,27 & 2,00 & 3,00 & 3,00 & 3,00 & 3,00 & 27 \\
\hline Diferença (Pós- Pré) & 0,63 & 0,97 & 0,00 & 3,00 & 0,00 & 0,00 & 1,00 & 27
\end{tabular}

3.2 A anotação está clara e objetiva, sem rasuras.

\begin{tabular}{lllllllll} 
Pré & 2,29 & 0,76 & 1,00 & 3,00 & 2,00 & 2,00 & 3,00 & 28 \\
Pós & 2,64 & 0,87 & 0,00 & 3,00 & 3,00 & 3,00 & 3,00 & 28 \\
\hline Diferença (Pós- Pré) & 0,36 & 1,03 & $-2,00$ & 2,00 & 0,00 & 0,00 & 1,00 & 28
\end{tabular}

3.3 A anotação está coerente com aos itens checados na Prescrição do enfermeiro.

Pré

Diferença (Pós- Pré)

\begin{tabular}{|c|c|c|c|c|c|c|}
\hline ,00 & 1,05 & 0,00 & 3,00 & 1,00 & 2,00 & 3,00 \\
\hline 00 & 0,00 & 3,00 & 3,00 & 3,00 & 3,00 & 3,00 \\
\hline & 1,05 & 0,00 & 3,00 & 0,00 & 1,00 & 2,00 \\
\hline
\end{tabular}

3.4 Anotação de enfermagem sem espaço em branco

\begin{tabular}{|llllllllll} 
Pré & 2,86 & 0,45 & 1,00 & 3,00 & 3,00 & 3,00 & 3,00 & 28 \\
\hline Pós & 3,00 & 0,00 & 3,00 & 3,00 & 3,00 & 3,00 & 3,00 & 28 & \\
Diferença (Pós- Pré) & 0,14 & 0,45 & 0,00 & 2,00 & 0,00 & 0,00 & 0,00 & 28
\end{tabular}

3.5. As anotações de enfermagem estão precedidas com o respectivo registro do horário em que a Intercorrência e/ou intervenção aconteceu.

\begin{tabular}{|lllllllll|l|}
\hline Pré & 2,96 & 0,19 & 2,00 & 3,00 & 3,00 & 3,00 & 3,00 & 27 \\
\hline Pós & 3,00 & 0,00 & 3,00 & 3,00 & 3,00 & 3,00 & 3,00 & 27 \\
\hline Diferença (Pós- Pré) & 0,04 & 0,19 & 0,00 & 1,00 & 0,00 & 0,00 & 0,00 & 27
\end{tabular}

O treinamento foi agendado em diversos horários, mas todos no horário de trabalho, e isso podem ser que tenha interferido nos resultados deste item.

$O$ resultado referente ao material didático poderia ter sido diferente se fosse utilizada a impressão colorida, vídeos ou se fosse disponibilizado o conteúdo para ser acessado com o uso da internet.

De acordo com Kirkpatrick, é importante obter uma avaliação de reação positiva, a fim de garantir a continuidade dos programas $^{(14)}$. A avaliação de aprendizagem foi aplicada em um estudo e mostrou que o melhor desempenho foi atingido pelos profissionais com maior tempo de experiência, o que demonstra que a presença de conhecimento prévio facilita a 
assimilação de um novo conhecimento ${ }^{(18)}$.

Observou-se que 7 (25,0\%) dos profissionais apresentaram acertos nos itens 6, 10 e 12, permanecendo em níveis similares mesmo após o treinamento.

O resultado destas três questões é relevante, pois em um recente estudo foi reforçado que os objetivos das anotações de enfermagem são garantir a comunicação efetiva entre a equipe de saúde e servir de documento legal de defesa dos profissionais, devendo ser realizada imediatamente após a observação de intercorrências, sendo precedida de data e hora ${ }^{(19)}$.

Com o resultado da avaliação de comportamento identifica-se a necessidade de promover uma nova sensibilização para reforçar a importância de realizar a anotação de enfermagem, logo no início do plantão.

As anotações de enfermagem devem ser realizadas após o cuidado prestado, orientação fornecida ou informação obti$\mathrm{da}^{(18)}$, porque se o profissional deixar para anotar no final do plantão ou após muito tempo das ações, poderá ocorrer o esquecimento de dados importantes.

A mudança de comportamento se refere à transferência efetiva do que foi aprendido no Programa de Treinamento e o que se exige do comportamento do treinando no trabalho( $\left.{ }^{2} 0\right)$. Por esse motivo é relevante a presença do enfermeiro em todos os treinamentos ministrados a sua equipe.

Para que ocorra mudança de comportamento, são necessárias quatro condições: o treinando precisa querer mudar, precisa saber o que precisa para mudar e como fazê-lo; precisa trabalhar no ambiente adequado e; precisa ser recompensado pela mudança ${ }^{(14)}$.

Segundo um estudo, se os lideres compreenderem os objetivos do treinamento e derem suporte para a transferência de aprendizagem, os resultados dos treinamentos tenderão a ser mais efeti$\operatorname{vos}^{(21)}$. O papel do enfermeiro inicia-se desde a elaboração da escala para participação e comunicação a equipe referente o tema, finalidade, data e horário do treinamento.
Os resultados obtidos nesta pesquisa convergem com um estudo sobre anotação de enfermagem que conclui ser necessário qualificar essa prática sensibilizando os profissionais de enfermagem sobre a sua importância ${ }^{(22)}$.

Outra pesquisa que fez analise da qualidade das anotações de enfermagem na divisão materno-infantil apresentou resultados satisfatórios como estes apresentados nesta pesquisa ${ }^{(23)}$.

Nas Instituições hospitalares ocorrem com frequência treinamentos para abordar o tema anotação de enfermagem, devido a necessidade de sensibilizar a equipe, que os registros realizados de forma adequada são fundamentais para o respaldo legal do profissional. Diante disso, torna-se motivador após a aplicação dessas ações, avaliar se houve aprendizado e mudança de comportamento.

\section{CONCLUSÃO} precisa saber 0 que precisa para mudar e como fazê-lo; precisa trabalhar no ambiente adequado e; precisa ser recompensado pela mudança ${ }^{(14)}$.

O grande desafio dos serviços responsáveis pelo desenvolvimento de treinamentos nas Instituições hospitalares não é somente fazer com que ocorra o treinamento, mas principalmente, mostrar que o treinamento foi eficaz e efetivo.

Este estudo teve como limitações a quantidade reduzida de sujeitos, a ausência de pré-teste e o curto intervalo de tempo entre as três observações na avaliação de comportamento.

Uma sugestão de estudos futuros refere-se à formulação de um instrumento de avaliação de comportamento com itens não relacionados ao conteúdo do treinamento, mas sim referente ao comportamento do participante ao voltar para o seu local de trabalho a fim de detectar mudanças. E esse instrumento deverá ser aplicado logo após o treinamento e mensalmente por três vezes após o treinamento.

Outra sugestão refere-se à reaplicação dos instrumentos validados em diferentes Instituições públicas e privadas, com valores e culturas distintas 
das pesquisadas nesta Instituição do estudo, de modo a conferir a abrangência e consistência ao resultado apresentado.

O resultado deste estudo demonstrou a fragilidade do comportamento dos profissionais de enfermagem que sabem, mas não realizam o que deve ser feito.

Frente a esta situação, considera-se necessário a responsabilização dos enfermeiros no processo de desenvolvimento da equipe em conjunto do Serviço de Educação Continuada, desde o levantamento das necessidades de treinamentos, no planejamento, na participação dos treinamentos, na supervisão, no monitoramento do desempenho da equipe e principalmente na análise do impacto da eficácia e da efetividade da assistência prestada ao cliente.

\section{Referências}

1. Bastos LF, Ciampone MH, Mira VL. Assessment of evaluation of transference support and training impact on the work of nurses. Rev Lat Am Enferm [Internet]. 2013 Nov-Dec [cited 2016 Dec 15];21(6):1274-81. Available from: http://www.scielo.br/pdf/rlae/ v21n6/0104-1169-rlae-21-06-01274.pdf

2. Chiavenato I. Gestão de pessoas. 3. ed. Rio de Janeiro: Elsevier; 2010.

3. Chiavenato I. Planejamento estratégico de gestão de pessoas. In: Chiavenato I, editor. Gestão de pessoas: o novo papel dos recursos humanos nas organizações. $4 a$ ed. São Paulo: Manole; 2014. p. 58.

4. Zerbini T, Abbad G. [Construction and validation of a training transfer scale]. Psicol Cienc Prof [Internet]. 2010 [cited 2016 Dec 12];30(4):684-97. Portuguese. Available from: http://www.scielo. $\mathrm{br} / \mathrm{pdf} / \mathrm{pcp} / \mathrm{v} 30 \mathrm{n} 4 / \mathrm{v} 30 \mathrm{n} 4 \mathrm{a} 02 . \mathrm{pdf}$

5. Oliveira IJ, Cruz CA. A importância da aplicação do treinamento e desenvolvimento nas organizações. Rev Cient ITPAC [Internet]. 2013 Apr [cited 2016 Dec 12];6(2):6. Available from: http:// www.itpac.br/arquivos/Revista/62/2.pdf

6. Gonçalves A, Mourão L. A expectativa em relação ao treinamento influencia o impacto das ações de capacitação? Rev Adm Pública [Internet]. 2011 Mar-Apr [cited 2016 Dec 13];45(2):483513. Available from: $\mathrm{http}: / / \mathrm{www} . \mathrm{scielo.br/pdf} / \mathrm{rap} / \mathrm{v} 45 \mathrm{n} 2 / 09 . \mathrm{pdf}$ 7. Scorsolini-Comin F, Inocente DF, Miura IK. [Evaluation of a corporate training program: in search of organizational change]. Rev Bras Orientac Prof [Internet]. 2012 [cited 2016 Dec 13];13(1):7586. Portuguese. Available from: http://pepsic.bvsalud.org/pdf/ rbop/v13n1/09.pdf

8. Chiavenato I. Treinamento e desenvolvimento de recursos humanos. 7a ed. São Paulo: Manole; 2009. Subsistema de desenvolvimento de recursos humanos; Capítulo 2.

9. Mira VL, Peduzzi M, Melleiro MM, Tronchin DM, Prado MF, Santos PT, et al. Analysis of the learning evaluation process of nursing staff actions. Rev Esc Enferm USP [Internet]. 2011 Dec [cited 2016 Dec 15];45 Spec No:1574-81. Available from: http://www. scielo.br/pdf/reeusp/v45nspe/en_v45nspea06.pdf

10. Tavares JC, Souza LB, Fogaça N. Levantamento bibliométrico sobre avaliação de treinamento no trabalho: cenário dos estudos publicados em periódicos da área de Administração entre 2009 e 2013. In: $38^{\circ}$ Encontro da ANPAD [Internet]; 2014 Sep 13-17; Rio de Janeiro. Rio de Janeiro: Associação Nacional de Pós-Graduação e Pesquisa em Administração; 2014 [cited 2016 Dec 15]. Available from: http://www.anpad.org.br/admin/pdf/2014_EnANPAD_ GPR1270.pdf

11. Coelho Junior FA, Abbad GS. [Construction and validation of a scale of the impact's measurement depth of a training in the distance in the performance in an organization of the brazilian banking sector]. REAd [Internet]. 2010 Jan-Apr [cited 2016 Dec 13];16(1):91-119. Portuguese. Available from: http://seer.ufrgs. br/index.php/read/article/view/38978/25012

12. Costa DB, Garcia SD, Vannuchi MT, Haddad MC. Impact of staff training in the health working process: an integrative re- view. J Nurs UFPE On-Line [Internet]. 2015 Apr [cited 2016 Dec 15];9(4):7439-47. Available from: http://www.revista.ufpe.br/revistaenfermagem/index.php/revista/article/view/6555/pdf_7575 13. Gonçalves VL. Avaliação de programas de treinamento e desenvolvimento da equipe de enfermagem de dois hospitais do município de São Paulo [tese de Livre Docência]. São Paulo: Universidade de São Paulo, Escola de Enfermagem; 2010.

14. Kirkpatrick DL, Kirkpatrick JD. Como avaliar programas de treinamento de equipes: os quatro níveis. Rio de Janeiro: Senac; 2010. 388 p.

15. Pereira JM. Manual de metodologia cientifica. 2a ed. São Paulo: Atlas; 2012.

16. Bastos LF. Avaliação da reação, aprendizagem e impacto de treinamento em um hospital do município de São Paulo [dissertação]. São Paulo: Universidade de São Paulo, Escola de Enfermagem; 2012.

17. da Costa DB, Vannuchi MT, Haddad M do C, Cardoso MG, da Silva LG, Garcia SD. [The cost of continuing education for the nursing team of a public university hospital]. Rev Eletr Enferm [Internet]. 2012 Apr-Jun [cited 2017 Jan 11];14(2):257-66. Portuguese. Available from: https://www.revistas.ufg.br/fen/article/ view/14540/11621

18. Chiavenato I. Planejamento estratégico de gestão de pessoas. In: Chiavenato I, editor. Gestão de pessoas: o novo papel dos recursos humanos nas organizações. 4a ed. São Paulo: Manole; 2014. p. 58.

19. Maxwell B. Documentação e informática. In: Potter PA, Perry AG, editors. Fundamentos de enfermagem. 7a ed. Rio de Janeiro: Elsevier; 2009. p. 384-94.

20. Scorsolini-Comin F, Inocente DF, Miura IK. [Evaluation of training, development and education programs in the organizational context: models and perspectives]. Rev Psicol: Org Trab [Internet]. 2011 Jan-Jun [cited 2016 Dec 13];11(1):37-53. Portuguese. Available from: https://periodicos.ufsc.br/index.php/rpot/article/ view/22245

21. Silva NS, Mourão L. [The influence of leadership styles of the results of training]. Estud Pesqui Psicol [Internet]. 2015 [cited 2016 Dec 18];15(1):260-83. Portuguese. Available from: http:// pepsic.bvsalud.org/pdf/epp/v15n1/v15n1a15.pdf

22. Jesus LR, Seignemartin BA, Vergílio MS, Silva EM. Anotação de enfermagem: retrato da prática cotidiana da enfermagem de um pronto atendimento. In: $17^{\circ}$ SENPE Seminário Nacional Pesquisa em Enfermagem [Internet]; 2013 Jun 3-5, Natal (RN). Brasília: Associação Brasileira de Enfermagem; 2013 [cited 2016 Dec 19]. p. 318-320. Available from: http://www.abeneventos.com.br/ anais_senpe/17senpe/pdf/0151co.pdf

23. Borsato FG, Rossaneis MA, Haddad MC, Vannuchi MT, Vituri DW. Assessment of quality of nursing documentation in a University Hospital. Acta Paul Enferm [Internet]. 2011 [cited 2016 Dec 19];24(4):527-33. Available from: http://www.scielo.br/pdf/ape/ v24n4/en_a13v24n4. 